\title{
Optimal inequalities for bounding Toader mean by arithmetic and quadratic means
}

Tie-Hong Zhao ${ }^{1}$, Yu-Ming Chu ${ }^{1 *}$ and Wen Zhang ${ }^{2}$

\section{"Correspondence:}

chuyuming2005@126.com

'School of Mathematics and

Computation Sciences, Hunan City

University, Yiyang, 413000, China

Full list of author information is

available at the end of the article

\section{Abstract}

In this paper, we present the best possible parameters $\alpha(r)$ and $\beta(r)$ such that the double inequality

$$
\begin{aligned}
{\left[\alpha(r) A^{r}(a, b)+(1-\alpha(r)) Q^{r}(a, b)\right]^{1 / r} } & <\operatorname{TD}[A(a, b), Q(a, b)] \\
& <\left[\beta(r) A^{r}(a, b)+(1-\beta(r)) Q^{r}(a, b)\right]^{1 / r}
\end{aligned}
$$

holds for all $r \leq 1$ and $a, b>0$ with $a \neq b$, and we provide new bounds for the complete elliptic integral $\mathcal{E}(r)=\int_{0}^{\pi / 2}\left(1-r^{2} \sin ^{2} \theta\right)^{1 / 2} d \theta(r \in(0, \sqrt{2} / 2))$ of the second kind, where $\operatorname{TD}(a, b)=\frac{2}{\pi} \int_{0}^{\pi / 2} \sqrt{a^{2} \cos ^{2} \theta+b^{2} \sin ^{2} \theta} d \theta, A(a, b)=(a+b) / 2$ and $Q(a, b)=\sqrt{\left(a^{2}+b^{2}\right) / 2}$ are the Toader, arithmetic, and quadratic means of $a$ and $b$, respectively.

MSC: 26E60

Keywords: arithmetic mean; Toader mean; quadratic mean; complete elliptic integral

\section{Introduction}

For $p \in[0,1], q \in \mathbb{R}$ and $a, b>0$ with $a \neq b$, the $p$ th generalized Seiffert mean $S_{p}(a, b), q$ th Gini mean $G_{q}(a, b), q$ th power mean $M_{q}(a, b)$, qth Lehmer mean $L_{q}(a, b)$, harmonic mean $H(a, b)$, geometric mean $G(a, b)$, arithmetic mean $A(a, b)$, quadratic mean $Q(a, b)$, Toader mean $T D(a, b)[1]$, centroidal mean $\bar{C}(a, b)$, contraharmonic mean $C(a, b)$ are, respectively, defined by

$$
\begin{aligned}
& S_{p}(a, b)= \begin{cases}\frac{p(a-b)}{\arctan [2 p(a-b) /(a+b)]}, & 0<p \leq 1, \\
(a+b) / 2, & p=0,\end{cases} \\
& G_{q}(a, b)= \begin{cases}{\left[\left(a^{q-1}+b^{q-1}\right) /(a+b)\right]^{1 /(q-2)},} & q \neq 2, \\
\left(a^{a} b^{b}\right)^{1 /(a+b)}, & q=2,\end{cases} \\
& M_{q}(a, b)= \begin{cases}{\left[\left(a^{q}+b^{q}\right) / 2\right]^{1 / q},} & q \neq 0, \\
\sqrt{a b}, & q=0,\end{cases}
\end{aligned}
$$

(c) The Author(s) 2017. This article is distributed under the terms of the Creative Commons Attribution 4.0 International License (http://creativecommons.org/licenses/by/4.0/), which permits unrestricted use, distribution, and reproduction in any medium, provided you give appropriate credit to the original author(s) and the source, provide a link to the Creative Commons license, and indicate if changes were made. 


$$
\begin{aligned}
& L_{q}(a, b)=\frac{a^{q+1}+b^{q+1}}{a^{q}+b^{q}}, \quad H(a, b)=\frac{2 a b}{a+b}, \quad G(a, b)=\sqrt{a b}, \\
& A(a, b)=\frac{a+b}{2}, \quad Q(a, b)=\sqrt{\frac{a^{2}+b^{2}}{2}}, \\
& T D(a, b)=\frac{2}{\pi} \int_{0}^{\pi / 2} \sqrt{a^{2} \cos ^{2} \theta+b^{2} \sin ^{2} \theta} d \theta, \\
& \bar{C}(a, b)=\frac{2\left(a^{2}+a b+b^{2}\right)}{3(a+b)}, \quad C(a, b)=\frac{a^{2}+b^{2}}{a+b} .
\end{aligned}
$$

It is well known that $S_{p}(a, b), G_{q}(a, b), M_{q}(a, b)$, and $L_{q}(a, b)$ are continuous and strictly increasing with respect to $p \in[0,1]$ and $q \in \mathbb{R}$ for fixed $a, b>0$ with $a \neq b$, and the inequalities

$$
\begin{aligned}
H(a, b) & =M_{-1}(a, b)=L_{-1}(a, b)<G(a, b)=M_{0}(a, b)=L_{-1 / 2}(a, b) \\
& <A(a, b)=M_{1}(a, b)=L_{0}(a, b)<T D(a, b)<\bar{C}(a, b) \\
& <Q(a, b)=M_{2}(a, b)<C(a, b)=L_{1}(a, b)
\end{aligned}
$$

hold for all $a, b>0$ with $a \neq b$.

The Toader mean $T D(a, b)$ has been well known in the mathematical literature for many years, it satisfies

$$
T D(a, b)=R_{E}\left(a^{2}, b^{2}\right)
$$

where

$$
R_{E}(a, b)=\frac{1}{\pi} \int_{0}^{\infty} \frac{[a(t+b)+b(t+a)] t}{(t+a)^{3 / 2}(t+b)^{3 / 2}} d t
$$

stands for the symmetric complete elliptic integral of the second kind (see [2-4]), therefore it cannot be expressed in terms of the elementary transcendental functions.

Let $r \in(0,1), \mathcal{K}(r)=\int_{0}^{\pi / 2}\left(1-r^{2} \sin ^{2} \theta\right)^{-1 / 2} d \theta$ and $\mathcal{E}(r)=\int_{0}^{\pi / 2}\left(1-r^{2} \sin ^{2} \theta\right)^{1 / 2} d \theta$ be, respectively, the complete elliptic integrals of the first and second kind. Then $\mathcal{K}\left(0^{+}\right)=\mathcal{E}\left(0^{+}\right)=$ $\pi / 2, \mathcal{K}(r)$, and $\mathcal{E}(r)$ satisfy the derivatives formulas (see [5], Appendix E, p.474-475)

$$
\begin{aligned}
& \frac{d \mathcal{K}(r)}{d r}=\frac{\mathcal{E}(r)-\left(1-r^{2}\right) \mathcal{K}(r)}{r\left(1-r^{2}\right)}, \quad \frac{d \mathcal{E}(r)}{d r}=\frac{\mathcal{E}(r)-\mathcal{K}(r)}{r}, \\
& \frac{d[\mathcal{K}(r)-\mathcal{E}(r)]}{d r}=\frac{r \mathcal{E}(r)}{1-r^{2}},
\end{aligned}
$$

the values $\mathcal{K}(\sqrt{2} / 2)$ and $\mathcal{E}(\sqrt{2} / 2)$ can be expressed as (see [6], Theorem 1.7)

$$
\mathcal{K}\left(\frac{\sqrt{2}}{2}\right)=\frac{\Gamma^{2}(1 / 4)}{4 \sqrt{\pi}}=1.854 \ldots, \quad \mathcal{E}\left(\frac{\sqrt{2}}{2}\right)=\frac{4 \Gamma^{2}(3 / 4)+\Gamma^{2}(1 / 4)}{8 \sqrt{\pi}}=1.350 \ldots
$$


where $\Gamma(x)=\int_{0}^{\infty} t^{x-1} e^{-t} d t(\operatorname{Re} x>0)$ is the Euler gamma function, and the Toader mean $T D(a, b)$ can be rewritten as

$$
T D(a, b)= \begin{cases}2 a \mathcal{E}\left(\sqrt{1-(b / a)^{2}}\right) / \pi, & a \geq b, \\ 2 b \mathcal{E}\left(\sqrt{1-(a / b)^{2}}\right) / \pi, & a<b .\end{cases}
$$

Recently, the Toader mean $\operatorname{TD}(a, b)$ has been the subject of intensive research. Vuorinen [7] conjectured that the inequality

$$
T D(a, b)>M_{3 / 2}(a, b)
$$

holds for all $a, b>0$ with $a \neq b$. This conjecture was proved by Qiu and Shen [8], and Barnard, Pearce and Richards [9], respectively.

Alzer and Qiu [10] presented a best possible upper power mean bound for the Toader mean as follows:

$$
T D(a, b)<M_{\log 2 /(\log \pi-\log 2)}(a, b)
$$

for all $a, b>0$ with $a \neq b$.

Neuman [2], and Kazi and Neuman [3] proved that the inequalities

$$
\begin{aligned}
& \frac{(a+b) \sqrt{a b}-a b}{A G M(a, b)}<T D(a, b)<\frac{4(a+b) \sqrt{a b}+(a-b)^{2}}{8 A G M(a, b)} \\
& T D(a, b)<\frac{1}{4}\left(\sqrt{(2+\sqrt{2}) a^{2}+(2-\sqrt{2}) b^{2}}+\sqrt{(2+\sqrt{2}) b^{2}+(2-\sqrt{2}) a^{2}}\right)
\end{aligned}
$$

hold for all $a, b>0$ with $a \neq b$, where $\operatorname{AGM}(a, b)$ is the arithmetic-geometric mean of $a$ and $b$.

In [11-13], the authors presented the best possible parameters $\lambda_{1}, \mu_{1} \in[0,1]$ and $\lambda_{2}, \mu_{2}, \lambda_{3}, \mu_{3} \in \mathbb{R}$ such that the double inequalities $S_{\lambda_{1}}(a, b)<T D(a, b)<S_{\mu_{1}}(a, b)$, $G_{\lambda_{2}}(a, b)<T D(a, b)<G_{\mu_{2}}(a, b)$ and $L_{\lambda_{3}}(a, b)<T D(a, b)<L_{\mu_{3}}(a, b)$ hold for all $a, b>0$ with $a \neq b$.

Let $\lambda, \mu, \alpha, \beta \in(1 / 2,1)$. Then Chu, Wang and Ma [14], and Hua and Qi [15] proved that the double inequalities

$$
\begin{aligned}
& C[\lambda a+(1-\lambda) b, \lambda b+(1-\lambda) a]<T D(a, b)<C[\mu a+(1-\mu) b, \mu b+(1-\mu) a], \\
& \bar{C}[\alpha a+(1-\alpha) b, \alpha b+(1-\alpha) a]<T D(a, b)<\bar{C}[\beta a+(1-\beta) b, \beta b+(1-\beta) a]
\end{aligned}
$$

hold for all $a, b>0$ with $a \neq b$ if and only if $\lambda \leq 3 / 4, \mu \geq 1 / 2+\sqrt{\pi(4-\pi)} /(2 \pi), \alpha \leq 1 / 2+$ $\sqrt{3} / 4$ and $\beta \geq 1 / 2+\sqrt{12 / \pi-3} / 2$.

In [16-20], the authors proved that the double inequalities

$$
\begin{aligned}
& \alpha_{1} Q(a, b)+\left(1-\alpha_{1}\right) A(a, b)<T D(a, b)<\beta_{1} Q(a, b)+\left(1-\beta_{1}\right) A(a, b), \\
& Q^{\alpha_{2}}(a, b) A^{\left(1-\alpha_{2}\right)}(a, b)<T D(a, b)<Q^{\beta_{2}}(a, b) A^{\left(1-\beta_{2}\right)}(a, b), \\
& \alpha_{3} C(a, b)+\left(1-\alpha_{3}\right) A(a, b)<T D(a, b)<\beta_{3} C(a, b)+\left(1-\beta_{3}\right) A(a, b),
\end{aligned}
$$




$$
\begin{aligned}
& \frac{\alpha_{4}}{A(a, b)}+\frac{1-\alpha_{4}}{C(a, b)}<\frac{1}{T D(a, b)}<\frac{\beta_{4}}{A(a, b)}+\frac{1-\beta_{4}}{C(a, b)}, \\
& \alpha_{5} C(a, b)+\left(1-\alpha_{5}\right) H(a, b)<T D(a, b)<\beta_{5} C(a, b)+\left(1-\beta_{5}\right) H(a, b), \\
& \alpha_{6}[C(a, b)-H(a, b)]+A(a, b)<T D(a, b)<\beta_{6}[C(a, b)-H(a, b)]+A(a, b), \\
& \alpha_{7} \bar{C}(a, b)+\left(1-\alpha_{7}\right) A(a, b)<T D(a, b)<\beta_{7} \bar{C}(a, b)+\left(1-\beta_{7}\right) A(a, b), \\
& \frac{\alpha_{8}}{A(a, b)}+\frac{1-\alpha_{8}}{\bar{C}(a, b)}<\frac{1}{T D(a, b)}<\frac{\beta_{8}}{A(a, b)}+\frac{1-\beta_{8}}{\bar{C}(a, b)}, \\
& \alpha_{9} Q(a, b)+\left(1-\alpha_{9}\right) H(a, b)<T D(a, b)<\beta_{9} Q(a, b)+\left(1-\beta_{9}\right) H(a, b), \\
& \frac{\alpha_{10}}{H(a, b)}+\frac{1-\alpha_{10}}{Q(a, b)}<\frac{1}{T D(a, b)}<\frac{\beta_{10}}{H(a, b)}+\frac{1-\beta_{10}}{Q(a, b)}
\end{aligned}
$$

hold for all $a, b>0$ with $a \neq b$ if and only if $\alpha_{1} \leq 1 / 2, \beta_{1} \geq(4-\pi) /[(\sqrt{2}-1) \pi], \alpha_{2} \leq 1 / 2$, $\beta_{2} \geq 4-2 \log \pi / \log 2, \alpha_{3} \leq 1 / 4, \beta_{3} \geq 4 / \pi-1, \alpha_{4} \leq \pi / 2-1, \beta_{4} \geq 3 / 4, \alpha_{5} \leq 5 / 8, \beta_{5} \geq 2 / \pi$, $\alpha_{6} \leq 1 / 8, \beta_{6} \geq 2 / \pi-1 / 2, \alpha_{7} \leq 3 / 4, \beta_{7} \geq 12 / \pi-3, \alpha_{8} \leq \pi-3, \beta_{8} \geq 1 / 4, \alpha_{9} \leq 5 / 6, \beta_{9} \geq$ $2 \sqrt{2} / \pi, \alpha_{10} \leq 0$, and $\beta_{10} \geq 1 / 6$.

The main purpose of this paper is to present the best possible parameters $\alpha(r)$ and $\beta(r)$ such that the double inequality

$$
\begin{aligned}
{\left[\alpha(r) A^{r}(a, b)+(1-\alpha(r)) Q^{r}(a, b)\right]^{1 / r} } & <T D[A(a, b), Q(a, b)] \\
& <\left[\beta(r) A^{r}(a, b)+(1-\beta(r)) Q^{r}(a, b)\right]^{1 / r}
\end{aligned}
$$

holds for all $r \leq 1$ and $a, b>0$ with $a \neq b$.

\section{Lemmas}

In order to prove our main result we need two lemmas, which we present in this section.

Lemma 2.1 Let $p \in(0,1), t \in(0, \sqrt{2} / 2), \lambda=(2+\sqrt{2})[1-2 \mathcal{E}(\sqrt{2} / 2) / \pi]=0.478 \ldots$ and

$$
f(t)=\frac{\pi p}{2} \sqrt{1-t^{2}}+\frac{\pi}{2}(1-p)-\mathcal{E}(t)
$$

Then $f(t)<0$ for all $t \in(0, \sqrt{2} / 2)$ if and only if $p \geq 1 / 2$ and $f(t)>0$ for all $t \in(0, \sqrt{2} / 2)$ if and only if $p \leq \lambda$.

Proof It follows from (2.1) that

$$
\begin{aligned}
& f\left(0^{+}\right)=0, \\
& f\left(\frac{\sqrt{2}}{2}\right)=\frac{\pi}{2}\left(1-\frac{\sqrt{2}}{2}\right)(\lambda-p), \\
& f^{\prime}(t)=\frac{f_{1}(t)}{t \sqrt{1-t^{2}}},
\end{aligned}
$$

where

$$
f_{1}(t)=\sqrt{1-t^{2}}[\mathcal{K}(t)-\mathcal{E}(t)]-\frac{\pi p}{2} t^{2}, \quad f_{1}\left(0^{+}\right)=0,
$$




$$
\begin{aligned}
& f_{1}\left(\frac{\sqrt{2}}{2}\right)=\frac{\sqrt{2}}{2}\left[\mathcal{K}\left(\frac{\sqrt{2}}{2}\right)-\mathcal{E}\left(\frac{\sqrt{2}}{2}\right)\right]-\frac{\pi p}{4}, \\
& f_{1}^{\prime}(t)=\frac{t[2 \mathcal{E}(t)-\mathcal{K}(t)]}{\sqrt{1-t^{2}}}-\pi p t, \\
& f_{1}^{\prime}\left(0^{+}\right)=0, \\
& f_{1}^{\prime}\left(\frac{\sqrt{2}}{2}\right)=2 \mathcal{E}\left(\frac{\sqrt{2}}{2}\right)-\mathcal{K}\left(\frac{\sqrt{2}}{2}\right)-\frac{\sqrt{2} \pi p}{2}, \\
& f_{1}^{\prime \prime}(t)=\frac{\left(3-2 t^{2}\right) \mathcal{E}(t)-\left(2-t^{2}\right) \mathcal{K}(t)}{\left(1-t^{2}\right)^{3 / 2}-\pi p,} \\
& f_{1}^{\prime \prime}\left(0^{+}\right)=\pi\left(\frac{1}{2}-p\right), \\
& f_{1}^{\prime \prime}\left(\frac{\sqrt{2}}{2}\right)=\sqrt{2}\left[4 \mathcal{E}\left(\frac{\sqrt{2}}{2}\right)-3 \mathcal{K}\left(\frac{\sqrt{2}}{2}\right)\right]-\pi p, \\
& f_{1}^{\prime \prime \prime}(t)=-\frac{\left(1+t^{2}\right)[\mathcal{K}(t)-\mathcal{E}(t)]+t^{2} \mathcal{K}(t)}{t\left(1-t^{2}\right)^{5 / 2}}<0
\end{aligned}
$$

for all $t \in(0, \sqrt{2} / 2)$.

It follows from $(2.13)$ that $f_{1}^{\prime \prime}(t)$ is strictly decreasing on $(0, \sqrt{2} / 2)$.

We divide the proof into three cases.

Case $1 p \geq 1 / 2$. Then (2.11) leads to

$$
f_{1}^{\prime \prime}\left(0^{+}\right) \leq 0
$$

From (2.14) and the monotonicity of $f_{1}^{\prime \prime}(t)$ we clearly see that $f_{1}^{\prime}(t)$ is strictly decreasing on $(0, \sqrt{2} / 2)$. Therefore, $f(t)<0$ for all $t \in(0, \sqrt{2} / 2)$ follows easily from (2.2), (2.4), (2.5), (2.8), and the monotonicity of $f_{1}^{\prime}(t)$.

Case $20<p \leq \lambda$. Then from (2.11) and (2.12) together with $4 \mathcal{E}(\sqrt{2} / 2)-3 \mathcal{K}(\sqrt{2} / 2)=$ $-0.159 \ldots$ we clearly see that

$$
f_{1}^{\prime \prime}\left(0^{+}\right)>0, \quad f_{1}^{\prime \prime}\left(\frac{\sqrt{2}}{2}\right)<0 .
$$

It follows from (2.15) and the monotonicity of $f_{1}^{\prime \prime}(t)$ that there exists $t_{0} \in(0, \sqrt{2} / 2)$ such that $f_{1}^{\prime}(t)$ is strictly increasing on $\left(0, t_{0}\right]$ and strictly decreasing on $\left[t_{0}, \sqrt{2} / 2\right)$.

Let $\lambda^{*}=\frac{\sqrt{2}}{\pi}\left[2 \mathcal{E}\left(\frac{\sqrt{2}}{2}\right)-\mathcal{K}\left(\frac{\sqrt{2}}{2}\right)\right]=0.381 \ldots$ and $\lambda^{* *}=\frac{2 \sqrt{2}}{\pi}\left[\mathcal{K}\left(\frac{\sqrt{2}}{2}\right)-\mathcal{E}\left(\frac{\sqrt{2}}{2}\right)\right]=0.453 \ldots \mathrm{We}$ divide the proof into three subcases.

Subcase $2.10<p \leq \lambda^{*}$. Then (2.9) leads to

$$
f_{1}^{\prime}\left(\frac{\sqrt{2}}{2}\right) \geq 0
$$

It follows from (2.8) and (2.16) together with the piecewise monotonicity of $f_{1}^{\prime}(t)$ that

$$
f_{1}^{\prime}(t)>0
$$

for all $t \in(0, \sqrt{2} / 2)$. 
Therefore, $f(t)>0$ for all $t \in(0, \sqrt{2} / 2)$ follows easily from (2.2), (2.4), (2.5), and (2.17). Subcase $2.2 \lambda^{*}<p \leq \lambda^{* *}$. Then (2.6) and (2.9) lead to

$$
\begin{aligned}
& f_{1}\left(\frac{\sqrt{2}}{2}\right) \geq 0 \\
& f_{1}^{\prime}\left(\frac{\sqrt{2}}{2}\right)<0 .
\end{aligned}
$$

It follows from (2.8) and (2.19) together with the piecewise monotonicity of $f_{1}^{\prime}(t)$ that there exists $t_{1} \in(0, \sqrt{2} / 2)$ such that $f_{1}(t)$ is strictly increasing on $\left(0, t_{1}\right]$ and strictly decreasing on $\left[t_{1}, \sqrt{2} / 2\right)$.

Equation (2.5) and inequality (2.18) together with the piecewise monotonicity of $f_{1}(t)$ lead to the conclusion that

$$
f_{1}(t)>0
$$

for all $t \in(0, \sqrt{2} / 2)$.

Therefore, $f(t)>0$ for all $t \in(0, \sqrt{2} / 2)$ follows easily from (2.2) and (2.4) together with (2.20).

Subcase $2.3 \lambda^{* *}<p \leq \lambda$. Then (2.3), (2.6), and (2.9) lead to

$$
\begin{aligned}
f\left(\frac{\sqrt{2}}{2}\right) & \geq 0 \\
f_{1}\left(\frac{\sqrt{2}}{2}\right) & <0 \\
f_{1}^{\prime}\left(\frac{\sqrt{2}}{2}\right) & <2 \mathcal{E}\left(\frac{\sqrt{2}}{2}\right)-\mathcal{K}\left(\frac{\sqrt{2}}{2}\right)-\frac{\sqrt{2} \pi}{2} \lambda^{* *} \\
& <2 \mathcal{E}\left(\frac{\sqrt{2}}{2}\right)-\mathcal{K}\left(\frac{\sqrt{2}}{2}\right)-\frac{\sqrt{2} \pi}{2} \lambda^{*}=0 .
\end{aligned}
$$

It follows from (2.8) and (2.23) together with the piecewise monotonicity of $f_{1}^{\prime}(t)$ that there exists $t_{2} \in(0, \sqrt{2} / 2)$ such that $f_{1}(t)$ is strictly increasing on $\left(0, t_{2}\right]$ and strictly decreasing on $\left[t_{2}, \sqrt{2} / 2\right)$.

From (2.4), (2.5), and (2.22) together with the piecewise monotonicity of $f_{1}(t)$ we clearly see that there exists $t_{3} \in(0, \sqrt{2} / 2)$ such that $f(t)$ is strictly increasing on $\left(0, t_{3}\right]$ and strictly decreasing on $\left[t_{3}, \sqrt{2} / 2\right)$.

Therefore, $f(t)>0$ for all $t \in(0, \sqrt{2} / 2)$ follows easily from (2.2) and (2.21) together with the piecewise monotonicity of $f(t)$.

Case $3 \lambda<p<1 / 2$. Then (2.3), (2.6), (2.9), (2.11), and (2.12) lead to

$$
\begin{aligned}
& f\left(\frac{\sqrt{2}}{2}\right)<0 \\
& f_{1}\left(\frac{\sqrt{2}}{2}\right)<\frac{\sqrt{2}}{2}\left[\mathcal{K}\left(\frac{\sqrt{2}}{2}\right)-\mathcal{E}\left(\frac{\sqrt{2}}{2}\right)\right]-\frac{\pi \lambda^{* *}}{4}=0 \\
& f_{1}^{\prime}\left(\frac{\sqrt{2}}{2}\right)<2 \mathcal{E}\left(\frac{\sqrt{2}}{2}\right)-\mathcal{K}\left(\frac{\sqrt{2}}{2}\right)-\frac{\sqrt{2} \pi \lambda^{*}}{2}=0
\end{aligned}
$$




$$
\begin{aligned}
f_{1}^{\prime \prime}\left(0^{+}\right)>0 & \begin{aligned}
f_{1}^{\prime \prime}\left(\frac{\sqrt{2}}{2}\right) & <\sqrt{2}\left[4 \mathcal{E}\left(\frac{\sqrt{2}}{2}\right)-3 \mathcal{K}\left(\frac{\sqrt{2}}{2}\right)\right]-\pi \lambda^{*} \\
& =-2 \sqrt{2}\left[\mathcal{K}\left(\frac{\sqrt{2}}{2}\right)-\mathcal{E}\left(\frac{\sqrt{2}}{2}\right)\right]<0
\end{aligned}
\end{aligned}
$$

It follows from (2.27) and (2.28) together with the monotonicity of $f_{1}^{\prime \prime}(t)$ that there exists $t_{4} \in(0, \sqrt{2} / 2)$ such that $f_{1}^{\prime}(t)$ is strictly increasing on $\left(0, t_{4}\right]$ and strictly decreasing on $\left[t_{4}, \sqrt{2} / 2\right)$.

Equation (2.8) and inequality (2.26) together with the piecewise monotonicity of $f_{1}^{\prime}(t)$ lead to the conclusion that there exists $t_{5} \in(0, \sqrt{2} / 2)$ such that $f_{1}(t)$ is strictly increasing on $\left(0, t_{5}\right]$ and strictly decreasing on $\left[t_{5}, \sqrt{2} / 2\right)$.

From (2.4), (2.5), (2.25), and the piecewise monotonicity of $f_{1}(t)$ we clearly see that there exists $t_{6} \in(0, \sqrt{2} / 2)$ such that $f(t)$ is strictly increasing on $\left(0, t_{6}\right]$ and strictly decreasing on $\left[t_{6}, \sqrt{2} / 2\right)$.

Therefore, there exists $t_{7} \in(0, \sqrt{2} / 2)$ such that $f(t)>0$ for $t \in\left(0, t_{7}\right)$ and $f(t)<0$ for $t \in\left(t_{7}, \sqrt{2} / 2\right)$ follows from (2.2) and (2.24) together with the piecewise monotonicity of $f(t)$.

Lemma 2.2 Let $r \in \mathbb{R}, a, b>0$ with $1<b / a<\sqrt{2}, c_{0}=2 \mathcal{E}(\sqrt{2} / 2) / \pi=0.859 \ldots, c_{1}=\sqrt{2} / 2$, $\lambda(r)$ and $U(r ; a, b)$ be defined by

$$
\lambda(r)=\frac{1-c_{0}^{r}}{1-c_{1}^{r}} \quad(r \neq 0), \quad \lambda_{0}=\frac{\log c_{0}}{\log c_{1}},
$$

and

$$
U(r ; a, b)=\left[\lambda(r) a^{r}+(1-\lambda(r)) b^{r}\right]^{1 / r} \quad(r \neq 0), \quad U(0 ; a, b)=a^{\lambda_{0}} b^{1-\lambda_{0}},
$$

respectively. Then the function $r \mapsto U(r ; a, b)$ is strictly decreasing on $(-\infty, \infty)$.

Proof Let $x=b / a \in(1, \sqrt{2}), r \neq 0$, and

$$
V(r, x)=(1-\lambda(r)) \log x-(\log \lambda(r))^{\prime}
$$

Then from (2.29)-(2.31) one has

$$
\begin{aligned}
& \log U(r ; a, b)=\log a+\frac{1}{r} \log \left(\lambda(r)+(1-\lambda(r)) x^{r}\right), \\
& \frac{\partial \log U(r ; a, b)}{\partial r}=\frac{\lambda^{\prime}(r)\left(1-x^{r}\right)+(1-\lambda(r)) x^{r} \log x}{r\left(\lambda(r)+(1-\lambda(r)) x^{r}\right)}-\frac{\log \left(\lambda(r)+(1-\lambda(r)) x^{r}\right)}{r^{2}}, \\
& \left.\frac{\partial \log U(r ; a, b)}{\partial r}\right|_{x=1}=0, \\
& \lambda^{\prime}(r)=\frac{\left(c_{1}^{r}-1\right) c_{0}^{r} \log c_{0}-\left(c_{0}^{r}-1\right) c_{1}^{r} \log c_{1}}{\left(c_{1}^{r}-1\right)^{2}}, \\
& \left.\left(\lambda(r)+(1-\lambda(r)) x^{r}\right)\right|_{x=\sqrt{2}}=\frac{1-c_{0}^{r}}{1-c_{1}^{r}}+\left(1-\frac{1-c_{0}^{r}}{1-c_{1}^{r}}\right) \frac{1}{c_{1}^{r}}=\frac{c_{0}^{r}}{c_{1}^{r}},
\end{aligned}
$$




$$
\begin{aligned}
& \left.\frac{\lambda^{\prime}(r)\left(1-x^{r}\right)+(1-\lambda(r)) x^{r} \log x}{r\left(\lambda(r)+(1-\lambda(r)) x^{r}\right)}\right|_{x=\sqrt{2}}=\frac{1}{r} \log \frac{c_{0}}{c_{1}} \\
& \left.\frac{\partial \log U(r ; a, b)}{\partial r}\right|_{x=\sqrt{2}}=0, \\
& \frac{\partial^{2} \log U(r ; a, b)}{\partial x \partial r}=\frac{\lambda(r) x^{r-1}}{\left(\lambda(r)+(1-\lambda(r)) x^{r}\right)^{2}} V(r, x), \\
& V(r, 1)=\frac{\log \frac{1}{c_{1}}}{\left(\frac{1}{c_{1}}\right)^{r}-1}-\frac{\log \frac{1}{c_{0}}}{\left(\frac{1}{c_{0}}\right)^{r}-1}<0, \\
& V(r, \sqrt{2})=c_{0}^{r}\left(\frac{\log c_{1}}{c_{1}^{r}-1}-\frac{\log c_{0}}{c_{0}^{r}-1}\right)>0,
\end{aligned}
$$

where inequalities (2.35) and (2.36) hold due to $c_{0}>c_{1}$ and the function $t \mapsto \log t /\left(t^{r}-1\right)$ is strictly decreasing on $(0, \infty)$.

Note that $\lambda(r) \in(0,1)$ and the function $x \rightarrow V(r, x)$ is strictly increasing on $(1, \sqrt{2})$. Then (2.34)-(2.36) lead to the conclusion that there exists $x_{0} \in(1, \sqrt{2})$ such that the function $x \mapsto \partial \log U(r ; a, b) / \partial r$ is strictly decreasing on $\left(1, x_{0}\right)$ and strictly increasing on $\left(x_{0}, \sqrt{2}\right)$.

It follows from (2.32) and (2.33) together with the piecewise monotonicity of the function $x \mapsto \partial \log U(r ; a, b) / \partial r$ on the interval $(1, \sqrt{2})$ that

$$
\frac{\partial \log U(r ; a, b)}{\partial r}<0
$$

for all $a, b>0$ with $1<b / a<\sqrt{2}$.

Therefore, Lemma 2.2 follows from (2.37).

\section{Main result}

Theorem 3.1 Let $c_{0}=2 \mathcal{E}(\sqrt{2} / 2) / \pi=0.859 \ldots, c_{1}=\sqrt{2} / 2$ and $\lambda(r)$ be defined by (2.29). Then the double inequality

$$
\begin{aligned}
{\left[\alpha(r) A^{r}(a, b)+(1-\alpha(r)) Q^{r}(a, b)\right]^{1 / r} } & <T D[A(a, b), Q(a, b)] \\
& <\left[\beta(r) A^{r}(a, b)+(1-\beta(r)) Q^{r}(a, b)\right]^{1 / r}
\end{aligned}
$$

holds for all $r \leq 1$ and $a, b>0$ with $a \neq b$ if and only if $\alpha(r) \geq 1 / 2$ and $\beta(r) \leq \lambda(r)$, where $r=0$ is the limit value of $r \rightarrow 0$.

Proof We first prove that Theorem 3.1 holds for $r=1$.

Since $A(a, b)<T D[A(a, b), Q(a, b)]<Q(a, b)$ for all $a, b>0$ with $a \neq b$, and $A(a, b)$, $T D(a, b)$ and $Q(a, b)$ are symmetric and homogeneous of degree 1 , without loss of generality, we assume that $\alpha(1), \beta(1) \in(0,1)$ and $a>b$. Let $t=(a-b) / \sqrt{2\left(a^{2}+b^{2}\right)} \in(0, \sqrt{2} / 2)$ and $p \in(0,1)$. Then (1.1) and (1.2) lead to

$$
\begin{aligned}
& A(a, b)=Q(a, b) \sqrt{1-t^{2}}, \quad T D[A(a, b), Q(a, b)]=\frac{2}{\pi} Q(a, b) \mathcal{E}(t), \\
& p A(a, b)+(1-p) Q(a, b)-T D[A(a, b), Q(a, b)]=\frac{2}{\pi} Q(a, b) f(t),
\end{aligned}
$$

where $f(t)$ is defined as in Lemma 2.1. 
Therefore, Theorem 3.1 for $r=1$ follows easily from Lemma 2.1 and (3.1).

Next, let $r<1$ and $a, b>0$ with $a \neq b$, then it follows from Theorem 3.1 for $r=1$ that

$$
\frac{A(a, b)+Q(a, b)}{2}<T D[A(a, b), Q(a, b)]<\lambda(1) A(a, b)+(1-\lambda(1)) Q(a, b) .
$$

Note that

$$
\begin{aligned}
& 1<\frac{Q(a, b)}{A(a, b)}<\sqrt{2}, \\
& \frac{T D[A(a, b), Q(a, b)]}{\left[\frac{A^{r}(a, b)+Q^{r}(a, b)}{2}\right]^{1 / r}}=\frac{2^{1+1 / r}}{\pi} \frac{\mathcal{E}(t)}{\left[1+\left(1-t^{2}\right)^{r / 2}\right]^{1 / r}}, \\
& \frac{T D[A(a, b), Q(a, b)]}{\left[\lambda(r) A^{r}(a, b)+(1-\lambda(r)) Q^{r}(a, b)\right]^{1 / r}}=\frac{2}{\pi} \frac{\mathcal{E}(t)}{\left[\lambda(r)\left(1-t^{2}\right)^{r / 2}+1-\lambda(r)\right]^{1 / r}}, \\
& \lim _{t \rightarrow 0^{+}} \frac{2^{1+1 / r}}{\pi} \frac{\mathcal{E}(t)}{\left[1+\left(1-t^{2}\right)^{r / 2}\right]^{1 / r}}=\lim _{t \rightarrow \sqrt{2} / 2} \frac{2}{\pi} \frac{\mathcal{E}(t)}{\left[\lambda(r)\left(1-t^{2}\right)^{r / 2}+1-\lambda(r)\right]^{1 / r}}=1 .
\end{aligned}
$$

Therefore, Theorem 3.1 for $r<1$ follows from (3.2)-(3.6) and Lemma 2.2 together with the monotonicity of the function $r \mapsto\left[\left(a^{r}+b^{r}\right) / 2\right]^{1 / r}$.

Let $r=1$. Then Theorems 3.1 leads to Corollary 3.2 immediately.

Corollary 3.2 Let $\lambda=(2+\sqrt{2})[1-2 \mathcal{E}(\sqrt{2} / 2) / \pi]$. Then the double inequality

$$
\frac{\pi}{4} \sqrt{1-t^{2}}+\frac{\pi}{4}<\mathcal{E}(t)<\frac{\pi}{2} \lambda \sqrt{1-t^{2}}+\frac{\pi}{2}(1-\lambda)
$$

holds for all $t \in(0, \sqrt{2} / 2)$.

\section{Competing interests}

The authors declare that they have no competing interests.

\section{Authors' contributions}

All authors contributed equally to the writing of this paper. All authors read and approved the final manuscript.

\section{Author details}

${ }^{1}$ School of Mathematics and Computation Sciences, Hunan City University, Yiyang, 413000, China. ${ }^{2}$ Icahn School of Medicine at Mount Sinai, Friedman Brain Institute, New York, 10033, United States.

\section{Acknowledgements}

The research was supported by the Natural Science Foundation of China under Grants 61673169, 11371125, 11401191, and 61374086 .

Received: 28 November 2016 Accepted: 17 January 2017 Published online: 25 January 2017

\section{References}

1. Toader, G: Some mean values related to the arithmetic-geometric mean. J. Math. Anal. Appl. 218(2), 358-368 (1998)

2. Neuman, E: Bounds for symmetric elliptic integrals. J. Approx. Theory 122(2), 249-259 (2003)

3. Kazi, H, Neuman, E: Inequalities and bounds for elliptic integrals. J. Approx. Theory 146(2), 212-226 (2007)

4. Kazi, H, Neuman, E: Inequalities and bounds for elliptic integrals II. In: Special Functions and Orthogonal Polynomials. Contemp. Math., vol. 471, pp. 127-138. Amer. Math. Soc., Providence, RI (2008)

5. Anderson, GD, Vamanamurthy, MK, Vuorinen, M: Conformal Invariants, Inequalities, and Quasiconformal Maps. Wiley, New York (1997)

6. Borwein, JM, Borwein, PB: Pi and the AGM. Wiley, New York (1987)

7. Vuorinen, M: Hypergeometric functions in geometric function theory. In: Special Functions and Differential Equations, Madras, 1977, pp. 119-126. Allied Publ., New Delhi (1998)

8. Qiu, S-L, Shen, J-M: On two problems concerning means. J. Hangzhou Inst. Electron. Eng. 17(3), 1-7 (1997) (in Chinese) 
9. Barnard, RW, Pearce, K, Richards, KC: An inequality involving the generalized hypergeometric function and the arc length of an ellipse. SIAM J. Math. Anal. 31(3), 693-699 (2000)

10. Alzer, H, Qiu, S-L: Monotonicity theorems and inequalities for the complete elliptic integrals. J. Comput. Appl. Math. 172(2), 289-312 (2004)

11. Chu, Y-M, Wang, M-K, Qiu, S-L, Qiu, Y-F: Sharp generalized Seiffert mean bounds for Toader mean. Abstr. Appl. Anal. 2011, Article ID 605259 (2011)

12. Chu, Y-M, Wang, M-K: Inequalities between arithmetic-geometric, Gini, and Toader means. Abstr. Appl. Anal. 2012, Article ID 830585 (2012)

13. Chu, Y-M, Wang, M-K: Optimal Lehmer mean bounds for the Toader mean. Results Math. 61(3-4), 223-229 (2012)

14. Chu, Y-M, Wang, M-K, Ma, X-Y: Sharp bounds for Toader mean in terms of contraharmonic mean with applications. J. Math. Inequal. 7(2), 161-166 (2012)

15. Hua, Y, Qi, F: A double inequality for bounding Toader mean by the centroidal mean. Proc. Indian Acad. Sci. Math. Sci. 124(4), 527-531 (2014)

16. Chu, Y-M, Wang, M-K, Qiu, S-L: Optimal combination bounds of root-square and arithmetic means for Toader mean. Proc. Indian Acad. Sci. Math. Sci. 122(1), 41-51 (2012)

17. Song, Y-Q, Jiang, W-D, Chu, Y-M, Yan, D-D: Optimal bounds for Toader mean in terms of arithmetic and contraharmonic means. J. Math. Inequal. 7(4), 751-757 (2013)

18. Li, W-H, Zheng, M-M: Some inequalities for bounding Toader mean. J. Funct. Spaces Appl. 2013, Article ID 394194 (2013)

19. Hua, Y, Qi, F: The best bounds for Toader mean in terms of the centroidal and arithmetic means. Filomat 28(4), 775-780 (2014)

20. Sun, H, Chu, Y-M: Bounds for Toader mean by quadratic and harmonic means. Acta Math. Sci. 35A(1), 36-42 (2015) (in (hinese)

\section{Submit your manuscript to a SpringerOpen ${ }^{\ominus}$ journal and benefit from:}

- Convenient online submission

Rigorous peer review

- Immediate publication on acceptance

- Open access: articles freely available online

- High visibility within the field

- Retaining the copyright to your article 